\title{
Gamma-secretase inhibitor enhances the cytotoxic effect of bortezomib in multiple myeloma
}

\author{
Feng Chen ${ }^{1}$, Alexandra Pisklakova, Ming Li, Rachid Baz, Daniel M Sullivan, and Yulia \\ Nefedova ${ }^{2}$ \\ H. Lee Moffitt Cancer Center and Research Institute, Tampa, FL, 33612
}

\begin{abstract}
Background-Targeting of Notch signaling with $\gamma$-secretase inhibitors (GSIs) has been considered a promising strategy for the treatment of hematological malignancies including multiple myeloma (MM). Here we investigated whether the cytotoxic effect of bortezomib, an agent commonly used in MM, could be enhanced by the addition of a GSI.

Methods-MM cells were treated with GSI, bortezomib or the combination thereof. Apoptosis of MM cells, proteasome activity and Notch signaling activation were determined. The effect of the drug combination was also evaluated in MM cells transfected with the active domain of Notch-1.

Results-Using MM cell lines and primary MM cells isolated from the bone marrow of patients with MM we found a strong synergistic effect of bortezomib in combination with one of the GSIs studied. We next investigated the mechanism underlying this synergistic effect and determined that the effect of the drug combination was mainly dependent on the ability of the selected GSI to inhibit proteasome activity in MM cells.
\end{abstract}

Conclusion-Our study demonstrates that selected GSIs that inhibit proteasome activity may be successfully used in combination with bortezomib enhancing its anti-MM effect.

\section{Keywords}

gamma-secretase inhibitor; Notch inhibitor; bortezomib; multiple myeloma

\section{Introduction}

Signals conveyed by the components of the tumor microenvironment are critically important for the survival and growth of cancer cells. One of them, Notch signaling, has been implicated in the pathogenesis of a variety of solid and hematological tumors including multiple myeloma (MM) [1]. The Notch family of proteins is a group of 4 highly conserved receptors (Notch 1-4) that are expressed on the cell surface and directly regulate gene transcription. Notch signaling is activated upon interaction of Notch receptors with their ligands Jagged-1, Jagged-2 or Delta-like-1, Delta-like-3 and Delta-like-4 [2]. In bone

\footnotetext{
${ }^{2}$ Corresponding author: Yulia Nefedova, H. Lee Moffitt Cancer Center and Research Institute, 12902 Magnolia Dr, MRC 2067H, Tampa FL 33612, Phone: (813) 745-8454, Fax (813) 745-1328 julia.nefedova @ moffitt.org.

${ }^{1}$ Present address: Department of Hematology, Qilu Hospital and School of Medicine, Shandong University, Jinan, Shandong, 250012, P.R.China

Ethical Standards

Experiments comply with the current laws of the United States.

Conflict of interest

The authors declare that they have no conflict of interest.
} 
marrow, stromal cells have been shown to express Notch ligands while MM cells express both Notch receptors and ligands [3-5]. Binding of Notch to its ligand initiates two consequent proteolytic cleavages; the second cleavage occurs within the transmembrane domain of Notch and is mediated by a multisubunit protease complex possessing $\gamma$-secretase activity. As a result, the intracellular (IC) active domain of Notch is liberated and translocated to the nucleus where it binds the transcriptional repressor CFB-1 (RBP-Jx) converting it into a transcriptional activator. The targets of CBF-1/Notch-IC complex include genes of Hes and Hey families as well as cyclin D1, p21, NF- $\kappa B$ and others [6].

Proteolytic processing of Notch by $\gamma$-secretase is a necessary step in the activation of this family of proteins. The $\gamma$-secretase protease complex, therefore, has been considered as an attractive target for Notch inhibition with a class of compounds called $\gamma$-secretase inhibitors (GSIs) [7]. Of note, Notch proteins are not the only substrates for $\gamma$-secretase; several known substrates processed by this protease include $\beta$-amyloid precursor protein, syndecan and CD44 among others [8]. Although a variety of different GSI compounds are available now, only a limited number are able to inhibit Notch signaling. Using MM cells we have previously demonstrated that GSI-XII, a compound from Calbiochem, specifically inhibited Notch signaling and induced apoptosis in these cells.

Despite recent advances in developing new therapeutics, MM still remains an incurable malignancy. Rational combination of drugs is one of the strategies allowing a substantial increase in treatment efficacy. The goal of the present study was to investigate whether GSIs could enhance the effect of bortezomib, an effective chemotherapeutic drug commonly used in MM treatment [9]. Our data indicate that one of the compounds, GSI-XII, strongly synergizes with bortezomib in an anti-MM effect and that the effect of the drug combination is mainly attributed to the ability of this selected GSI to inhibit proteasome activity.

\section{Materials and Methods}

\subsection{Cell cultures and reagents}

Human MM NCI-H929, U266 and RPMI-8226 cell lines were obtained from ATCC and cultured as previously described [3]. GSI-XII (Z-IL-CHO) and GSI-IX (DAPT) were purchased from Calbiochem (San Diego, CA), edaravone purchased from Sigma, pancaspase inhibitor z-VAD-FMK obtained from Bachem (Torrance, CA), and bortezomib purchased from LC Laboratories (Woburn, MA). All of these compounds were dissolved in dimethyl sulfoxide. Unless indicated, the final concentration of GSI-XII was $3 \mu \mathrm{M}$, bortezomib $2-3 \mathrm{nM}$, and z-VAD-FMK $100 \mu \mathrm{M}$.

\subsection{Isolation of primary MM cells}

The collection of bone marrow samples from patients with MM was approved by the University of South Florida Institutional Review Board and informed consent was obtained from the patients. CD138 ${ }^{+}$primary MM cells were isolated from bone marrow aspirates using a magnetic beads cell separation technique (Miltenyi Biotec, Auburn, CA). Briefly, mononuclear cells were isolated from bone marrow aspirates using Ficoll-Paque gradient centrifugation and incubated with CD138-MicroBeads followed by magnetic separation of positive cells using the MidiMACS system according to the manufacturer's protocol (Miltenyi Biotec).

\subsection{Flow cytometry}

Apoptosis of MM cells was detected by an Annexin V binding assay using an LSR II flow cytometer (BD) as described previously [10]. Briefly, MM cells were collected, washed twice with ice cold PBS, once with binding buffer, and stained with Annexin V conjugated 
with FITC or APC and DAPI. Ten thousand events were acquired. Data were analyzed using FlowJo software (Tree Star, Inc, Ashland, OR).

\subsection{Cell transfection}

Vector pIRES2-AcGFP1 was purchased from Clontech (Mountain View, CA). The intracellular domain (IC) of Notch-1 was subcloned into the Sma I site of pIRES2-AcGFP1 vector. The presence of the insert and its orientation were confirmed by sequencing. U266 MM cells were transfected with vector pIRES2-AcGFP1-Notch1-IC or empty vector pIRES2-AcGFP1 using the nucleofector device (Amaxa-Lonza), buffer R and program T16.

\subsection{Quantitative real-time PCR}

MM cell lines were treated with DAPT for $24 \mathrm{hrs}$. Real-time PCR was performed as described earlier [3] using an ABI Prism 7900HT instrument. Hes-1 expression was normalized to the expression of the endogenous control gene 18S.

\subsection{Proteasome activity evaluation}

MM cells were plated in 96-well plates in triplicates and treated with GSI-XII, DAPT, bortezomib, or the drug combinations with or without $100 \mathrm{uM}$ edaravone for indicated period of time. After that chymotrypsin-like, trypsin-like or caspase-like proteasome activity was measured using a corresponding Proteasome-Glo cell-based assay kit (Promega, Madison, WI) according to the manufacturer's instructions. The luminescence signal was detected using a Synergy HT instrument (Bio Tek).

\subsection{Western blotting}

Western blotting was performed according to the standard protocol as described previously [3]. Membranes were blocked in 5\% milk for $1 \mathrm{hr}$ at room temperature and then incubated overnight at $4{ }^{\circ} \mathrm{C}$ with the primary antibodies against PARP, cleaved caspase 3 (Cell Signaling Technology, Danvers, MA), ubiquitinylated proteins (clone FK2, Millipore) and $\beta$-actin (Santa Cruz, Santa Cruz, CA). Membranes were then washed, incubated with the corresponding secondary antibodies, and developed using ECL.

\subsection{Statistical analysis}

The statistical significance of differences observed in cells treated with a single drug compared to cells treated with a drug combination was determined using the Student $t$ test. The $\mathrm{p}$ values less than 0.05 were considered significant.

\section{Results}

Initially, we determined a possible synergistic effect of the combination GSI-XII with bortezomib. To use each drug as a single agent we selected concentrations that induced apoptosis in only minimal proportion of cells. The combination of GSI-XII with bortezomib had a potent cytotoxic effect in all MM cell lines studied as well as in primary CD138 $\mathrm{MM}$ cells isolated from the bone marrow of six patients with MM (Fig. 1a,b). The effect of this drug combination was caspase-dependent, as confirmed by cleavage of caspase 3 and PARP (Fig. 1c). Pre-treatment of cells with $100 \mu \mathrm{M}$ of the pan-caspase inhibitor z-VAD-FMK completely abrogated the effect of the GSI-XII/bortezomib combination (data not shown).

Our previous data have demonstrated that inhibition of Notch signaling contributed to the cytotoxic effect of GSI-XII on MM cells [10]. In order to further investigate the involvement of Notch in the effect of the GSI-XII/bortezomib drug combination, we overexpressed the active IC domain of Notch-1 in MM cells using the bicistronic vector pIRES2-AcGFP1- 
Notch-1IC. The active form of Notch does not require proteolytic cleavage; therefore, its overexpression should overcome the GSI-XII effects mediated through inhibition of Notch. As anticipated, overexpression of the active form of Notch-1 substantially decreased apoptosis induced by the drug combination. However, it still did not abrogate the ability of GSI-XII and bortezomib to synergize in an anti-MM effect (Fig. 2), suggesting involvement of other mechanisms in the observed phenomenon.

We next evaluated the effect of another Notch inhibitor DAPT (GSI-IX, Calbiochem) in combination with bortezomib on MM cells. Initially, the effect of DAPT on Notch signaling was confirmed by measuring the expression of Notch downstream target gene Hes-1. Although at selected doses DAPT was able to inhibit Notch signaling (Fig. 3a) and induce apoptosis of MM cells, it did not enhance the cytotoxicity of bortezomib (Fig. 3b).

In an attempt to identify the mechanism by which GSI-XII could enhance the effect of bortezomib, we investigated the ability of this compound to inhibit proteasome activity. Three proteasome enzymatic activities that regulate degradation of proteins have been characterized including chymotrypsin-like, trypsin-like and caspase-like. GSI-XII was able to significantly inhibit all three of them with a more profound effect on chymotrypsin-like and caspase-like activities. At selected dose of $2 \mathrm{nM}$ bortezomib, a known proteasome inhibitor, substantially reduced chymotrypsin-like activity, but was not able to affect trypsinlike and caspase-like activities (Fig. 4a). Treatment of MM cell lines and primary CD138 $\mathrm{MM}$ cells isolated from the bone marrow of patients with MM with combination of these two compounds resulted in significant inhibition of chymotrypsin-like proteasome activity as compared to the cells treated with either single compound (Fig. 4a,b). However, no substantial effect of the drug combination was observed on trypsin-like or caspase-like activities due to the lack of the blocking ability of bortezomib on these proteasome activities (Fig. 4a). Thus, our data indicate that apoptosis of MM cells induced by combination of GSI-XII and bortezomib may be mediated by the inhibition of chymotrypsin-like rather than caspase-like or trypsin-like proteasome activity. Blocking proteasome activity and inability to break down intracellular proteins leads to accumulation of mono- and polyubiquitinylated proteins. In order to detect the level of ubiquitinylated proteins after treatment with bortezomib, GSI-XII or their combination Western blotting with antibody recognizing mono- and poly-ubiquitinylated proteins but not free ubiquitin was performed. As demonstrated on Fig 4c, inhibition of proteasome activity by bortezomib/GSI-XII drug combination resulted in substantial accumulation of ubiquitinylated proteins. In contrast to GSI-XII, no effect of DAPT on chymotrypsin-like proteasome activity was observed (Fig. $4 d)$.

It has been recently reported that edaravone, a free radical scavenger, could block the proteasome inhibitory activity of selected proteasome inhibitors [11]. Consistent with these data, we found that treatment of MM cells with edaravone reversed inhibition of chymotrypsin-like proteasome activity induced by GSI-XII and GSI-XII/bortezomib combination (Fig. 5a). Edaravone also completely abrogated the cytotoxic effect of the drug combination (Fig. 5b).

\section{Discussion}

The present study aimed to determine whether the cytotoxic anti-tumor effect of bortezomib, a chemotherapeutic drug commonly used for MM treatment, could be enhanced by the addition of a GSI/Notch inhibitor. Both GSIs tested inhibited Notch signaling in MM cells. However, they had distinct effects on MM cells viability when combined with bortezomib. One of them, GSI-XII, dramatically enhanced the cytotoxicity of bortezomib while the addition of another $\gamma$-secretase inhibitor, DAPT, did not change the proportion of MM cells 
undergoing apoptosis. Our results demonstrate that the distinct effects of two GSIs depended largely on the ability of the specific GSI to inhibit proteasome activity rather than Notch signaling.

The question whether the cytotoxicity of GSIs is mediated by the inhibition of Notch or proteasome activity has been recently a subject of several studies. Han and colleagues demonstrated the cytotoxic effect of GSI-I in a panel of 6 breast cancer cell lines [11]. However, treatment with DAPT or the $\gamma$-secretase inhibitor L-685,458 had no effect on the survival of these cells. The authors claimed that the observed cytotoxic effect of GSI-I was mediated by inhibition of proteasome activity; however, this compound also inhibited Notch signaling in the majority of cell lines studied. In another study, Rasul and colleagues demonstrated a potent cytotoxic effect of GSI-I in three breast cancer cell lines as well as in a NCI panel of 60 cancer cell lines [12]. The authors attributed this effect to the ability of GSI-I to inhibit $\gamma$-secretase activity, downregulate Notch signaling as well as inhibit proteasome activity in breast cancer cells. Thus, until now the relative contribution of the inhibition of proteasome activity and inhibition of Notch signaling in GSI-mediated apoptosis was unclear.

Our previous work had demonstrated that GSI-XII, when used at concentrations of 5-7 $\mu \mathrm{M}$, inhibited Notch signaling in MM cell lines. Furthermore, we observed a significant cytotoxic effect of this compound. In order to investigate whether the cytotoxicity of GSIXII was mediated through the Notch pathway, we overexpressed the Notch downstream target gene Hes-1 in MM cells using a bicistronic vector pIRES2-GFP-Hes1. Flow cytometry analysis of a gated GFP-positive population of cells demonstrated that overexpression of Hes-1 almost completely abrogated the cytotoxic effect of GSI-XII [13]. These data strongly indicate that the cytotoxic effect of selected compound is largely mediated through inhibition of Notch signaling. At the same time, a low proportion of GSIXII-treated cells overexpressing Hes-1 [10] or Notch1-IC (Fig. 2) were still undergoing apoptosis suggesting the existence of another mechanism involved in GSI-XII induced MM cell death. Low concentrations of GSI-XII $(3-4 \mu \mathrm{M})$ were sufficient to induce apoptosis in a small proportion of MM cells (below 5\%; Fig. 1a). At the same time a significant reduction in proteasome activity (Fig. 4a) was observed in these cells. These findings were consistent with recently reported data suggesting that selected GSI compounds like GSI-I (Calbiochem) could also function as proteasome inhibitors in breast cancer cell lines [11, 14]. Overexpression of the active domain of Notch-1 in MM cells did not abrogate the synergistic effect of the GSI-XII/bortezomib combination (Fig. 2) suggesting that this effect was Notch-independent.

In order to confirm that the cytotoxic effect of the GSI-XII/bortezomib combination depends on the inhibition of proteasome activity, we utilized another well known GSI/Notch inhibitor, DAPT. Treatment of MM cells with DAPT resulted in a dose-dependent inhibition of Notch and induction of apoptosis with no effect on proteasome activity (Fig. 3). As anticipated, the addition of bortezomib did not increase the proportion of DAPT-treated MM cells undergoing apoptosis (Fig. 3).

Taken together, our data indicate that GSIs could induce tumor cell apoptosis through two independent mechanisms: the inhibition of Notch signaling and inhibition of proteasome activity. However, the ability of the selected GSI to dramatically enhance the effect of bortezomib depends entirely on its proteasome inhibitory activity. Understanding the structural properties of GSI compounds and their molecular mechanisms will be critical for clinical use and the design of rational drug combinations. 


\section{Acknowledgments}

This work was supported by National Institute of Health/National Cancer Institute grant 1RO1 CA130923 (to Y.N.). This work has been supported in part by the Flow Cytometry Core Facility at the H. Lee Moffitt Cancer Center.

\section{References}

1. Leong K, Karsan A. Recent insights into the role of Notch signaling in tumorigenesis. Blood. 2006; 107:2223-2233. [PubMed: 16291593]

2. Ohishi K, Varnum-Finney B, Bernstein ID. The notch pathway: modulation of cell fate decisions in hematopoiesis. Int J Hematol. 2002; 75:449-459. [PubMed: 12095143]

3. Nefedova Y, Cheng P, Alsina M, Dalton W, Gabrilovich D. Involvement of Notch-1 signaling in bone marrow stroma-mediated de novo drug resistance of myeloma and other malignant lymphoid cell lines. Blood. 2004; 103:3503-3510. [PubMed: 14670925]

4. Jundt F, Probsting KS, Anagnostopoulos I, Muehlinghaus G, Chatterjee M, Mathas S, Bargou RC, Manz R, Stein H, Dorken B. Jagged1-induced Notch signaling drives proliferation of multiple myeloma cells. Blood. 2004; 103:3511-3515. [PubMed: 14726396]

5. Skrtić A, Korać P, Krišto D, Ajduković Stojisavljević R, Ivanković D, Dominis M. Immunohistochemical analysis of NOTCH1 and JAGGED1 expression in multiple myeloma and monoclonal gammopathy of undetermined significance. Hum Pathol. 2010; 41:1702-1710. [PubMed: 20800871]

6. Nefedova Y, Gabrilovich D. Mechanisms and clinical prospects of Notch inhibitors in the therapy of hematological malignancies. Drug Resist Updat. 2008; 11:210-218. [PubMed: 18951834]

7. Shih I-M, Wang T-L. Notch Signaling, gamma-Secretase Inhibitors, and Cancer Therapy. Cancer Res. 2007; 67:1879-1882. [PubMed: 17332312]

8. Beel A, Sanders C. Substrate specificity of g-secretase and other intramembrane proteases. Cell. Mol. Life Sci. 2008; 65:1311-1334. [PubMed: 18239854]

9. Shah J, Orlowski R. Proteasome inhibitors in the treatment of multiple myeloma. Leukemia. 2009; 23:1964-1979. [PubMed: 19741722]

10. Nefedova Y, Sullivan D, Bolick S, Dalton W, Gabrilovich D. Inhibition of Notch signaling induces apoptosis of myeloma cells and enhances sensitivity to chemotherapy. Blood. 2008; 111:22202229. [PubMed: 18039953]

11. Han J, Ma I, Hendzel M, Allalunis-Turner J. The cytotoxicity of gamma-secretase inhibitor I to breast cancer cells is mediated by proteasome inhibition, not by gamma-secretase inhibition. Breast Cancer Res. 2009; 11:R57. [PubMed: 19660128]

12. Rasul S, Balasubramanian R, Filipović A, Slade M, Yagüe E, Coombes R. Inhibition of gammasecretase induces G2/M arrest and triggers apoptosis in breast cancer cells. Br J Cancer. 2009; 100:1879-1888. [PubMed: 19513078]

13. Schwarzer R, Kaiser M, Acikgoez O, Heider U, Mathas S, Preissner R, Sezer O, Doerken B, Jundt F. Notch inhibition blocks multiple myeloma cell-induced osteoclast activation. Leukemia. 2008

14. Monticone M, Biollo E, Fabiano A, Fabbi M, Daga A, Romeo F, Maffei M, Melotti A, Giaretti W, Corte G, Castagnola P. z-Leucinyl-leucinyl-norleucinal induces apoptosis of human glioblastoma tumor-initiating cells by proteasome inhibition and mitotic arrest response. Mol Cancer Res. 2009; 7:1822-1834. [PubMed: 19861404] 

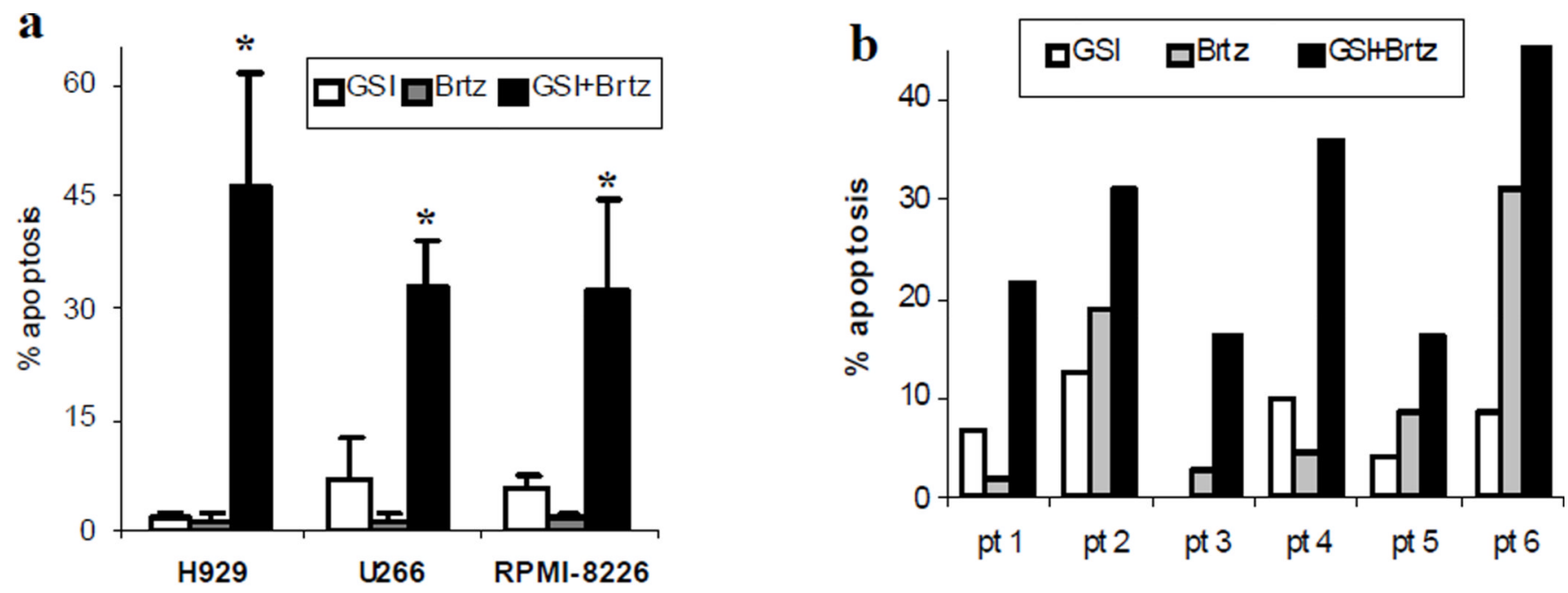

c $\quad \begin{array}{lllll} & v & B & G & B+G\end{array}$

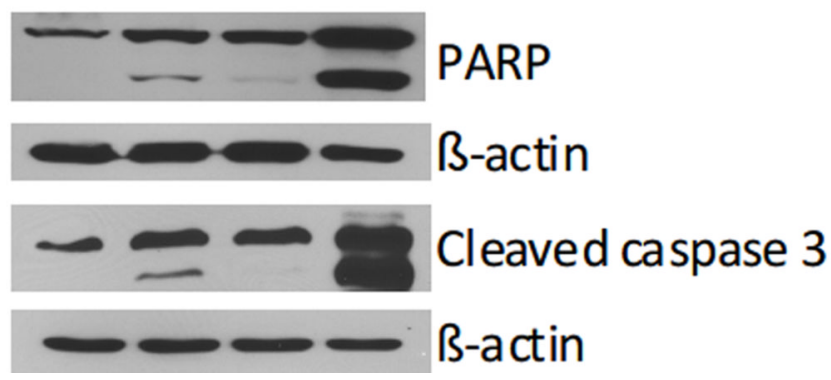

Fig. 1. Combined cytotoxic effect of GSI-XII and bortezomib in MM cells

Human MM cell lines (a,c) or primary CD138 ${ }^{+}$MM cells isolated from the bone marrow of six patients (pt) with MM (b) were treated for $24 \mathrm{hrs}$ with either $2 \mathrm{nM}$ (H929 cells) or $3 \mathrm{nM}$ bortezomib (Brtz), $3 \mu \mathrm{M}$ (for cell lines) and $5 \mu \mathrm{M}$ (for primary samples) GSI-XII (GSI) or the combination thereof. (a,b) Apoptosis of MM cells was evaluated by an Annexin V binding assay using flow cytometry. Values for specific drug-induced apoptosis (values of apoptosis in cells treated with vehicle control subtracted from values of apoptosis in cells treated with drugs) are shown. * - indicate a statistically significant difference $(\mathrm{p}<0.05)$ between the combination group and single drug-treated groups. For cell lines, at least three independent experiments which yielded the same results were performed. (c) H929 cells were collected and subjected to Western blotting with the indicated antibodies. Equal loading was confirmed by re-probing the membranes with antibody against beta-actin 


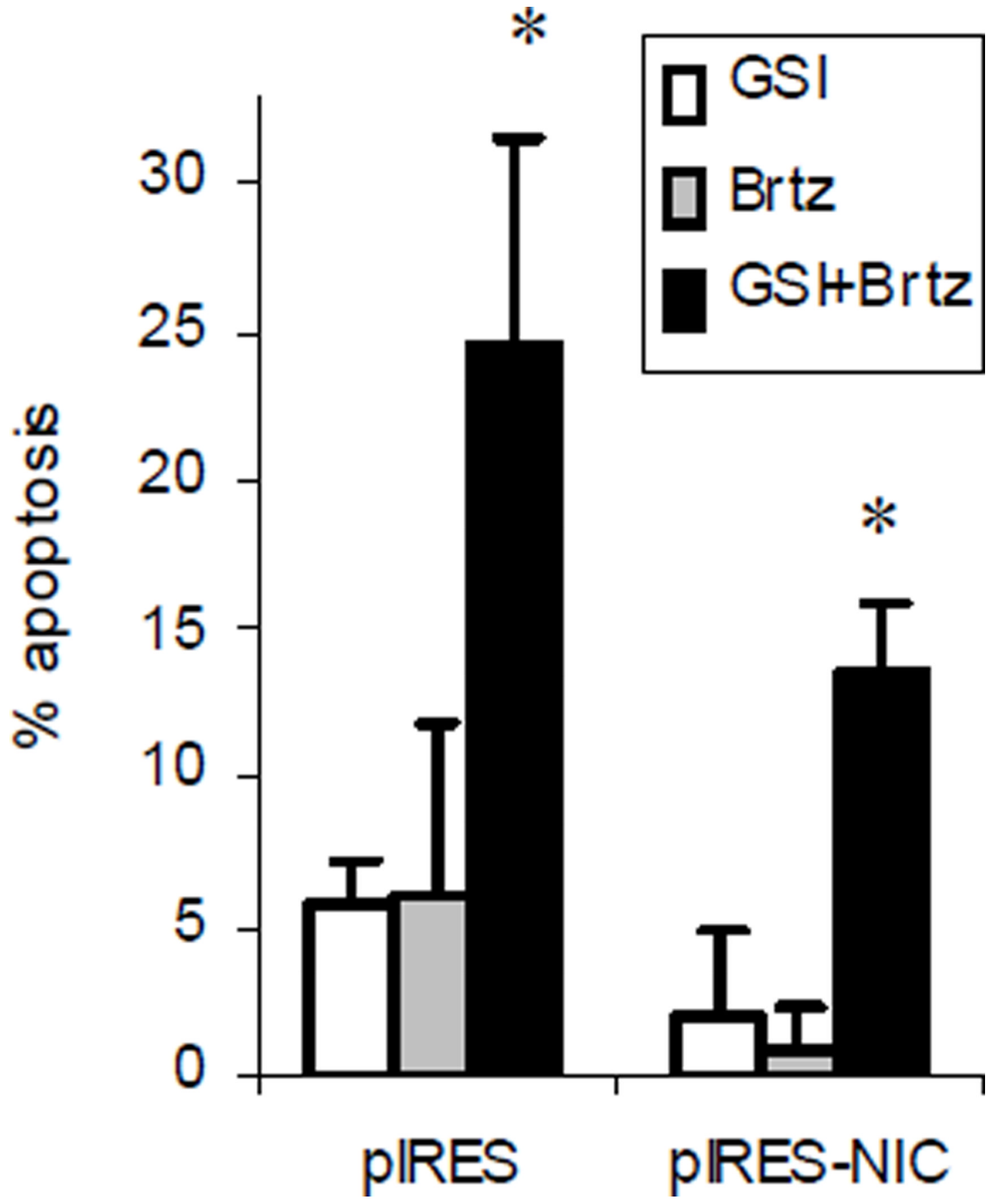

Fig. 2. Overexpression of the active form of Notch-1 does not abrogate the synergistic effect of a drug combination

MM U266 cells were transfected with either the pIRES2-AcGFP1 (pIRES) or pIRES2-

AcGFP1-Notch1-IC (pIRES-NIC) vector using the nucleofector device (Amaxa) followed by a $24 \mathrm{hr}$ treatment with GSI-XII, Brtz, or the drug combination. Apoptosis in a gated GFPpositive population of cells was detected using Annexin V-APC/DAPI staining using flow cytometry with a LSR II instrument (BD). The experiment was performed three times with similar results. * - indicate a statistically significant difference $(\mathrm{p}<0.05)$ between the combination group and single drug-treated groups 
$\mathbf{a}$

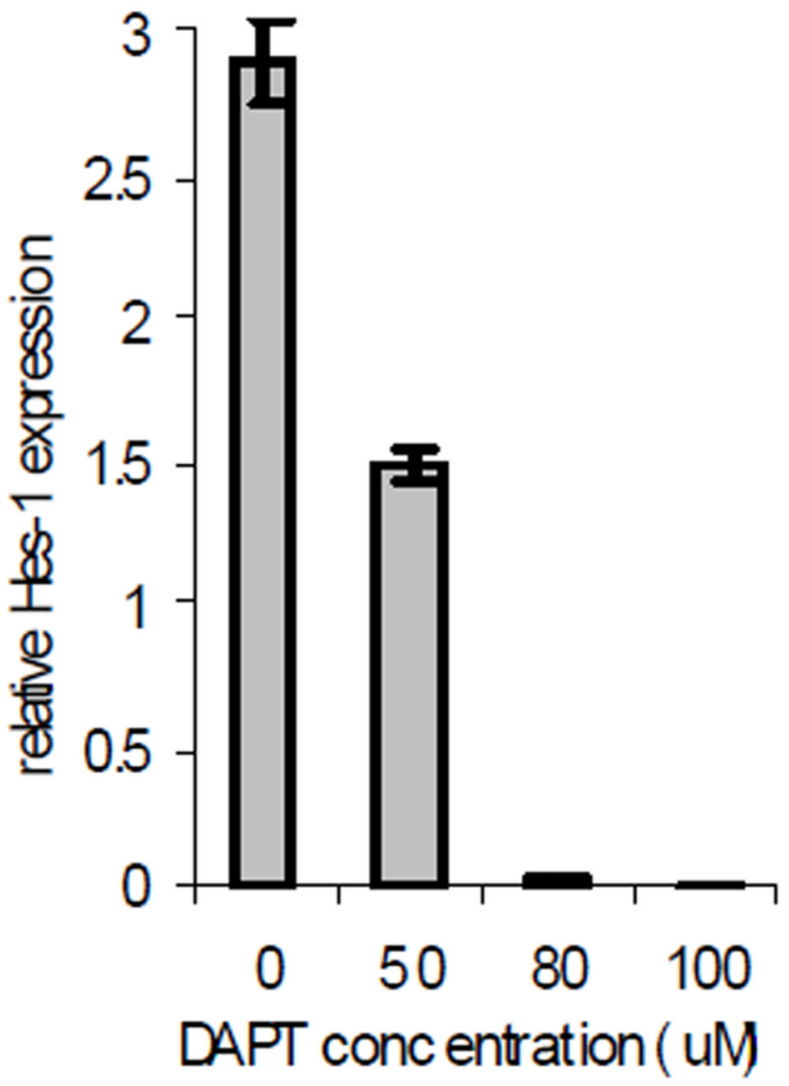

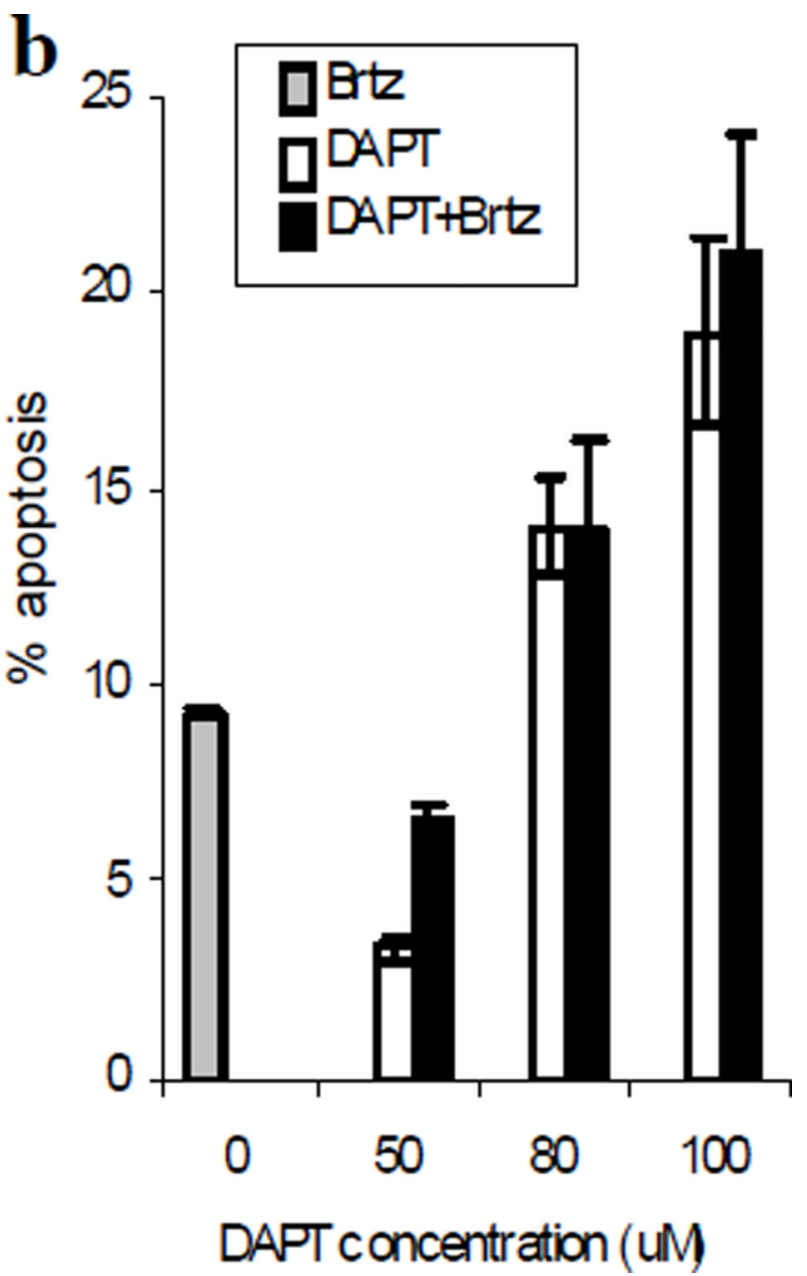

Fig. 3. Effect of DAPT on MM cells

H929 MM cells were treated with different concentrations of DAPT, $2 \mathrm{nM}$ bortezomib (Brtz), or the combination thereof for $24 \mathrm{hr}$. (a) The expression of the Hes-1 gene was evaluated by real-time PCR and normalized to the expression of the endogenous control gene 18S. (b) Apoptosis was evaluated by Annexin V-FITC/DAPI staining using a LSR II flow cytometer. Shown are mean $\pm \mathrm{SD}$ values of specific drug-induced apoptosis in two independent experiments performed in duplicate 

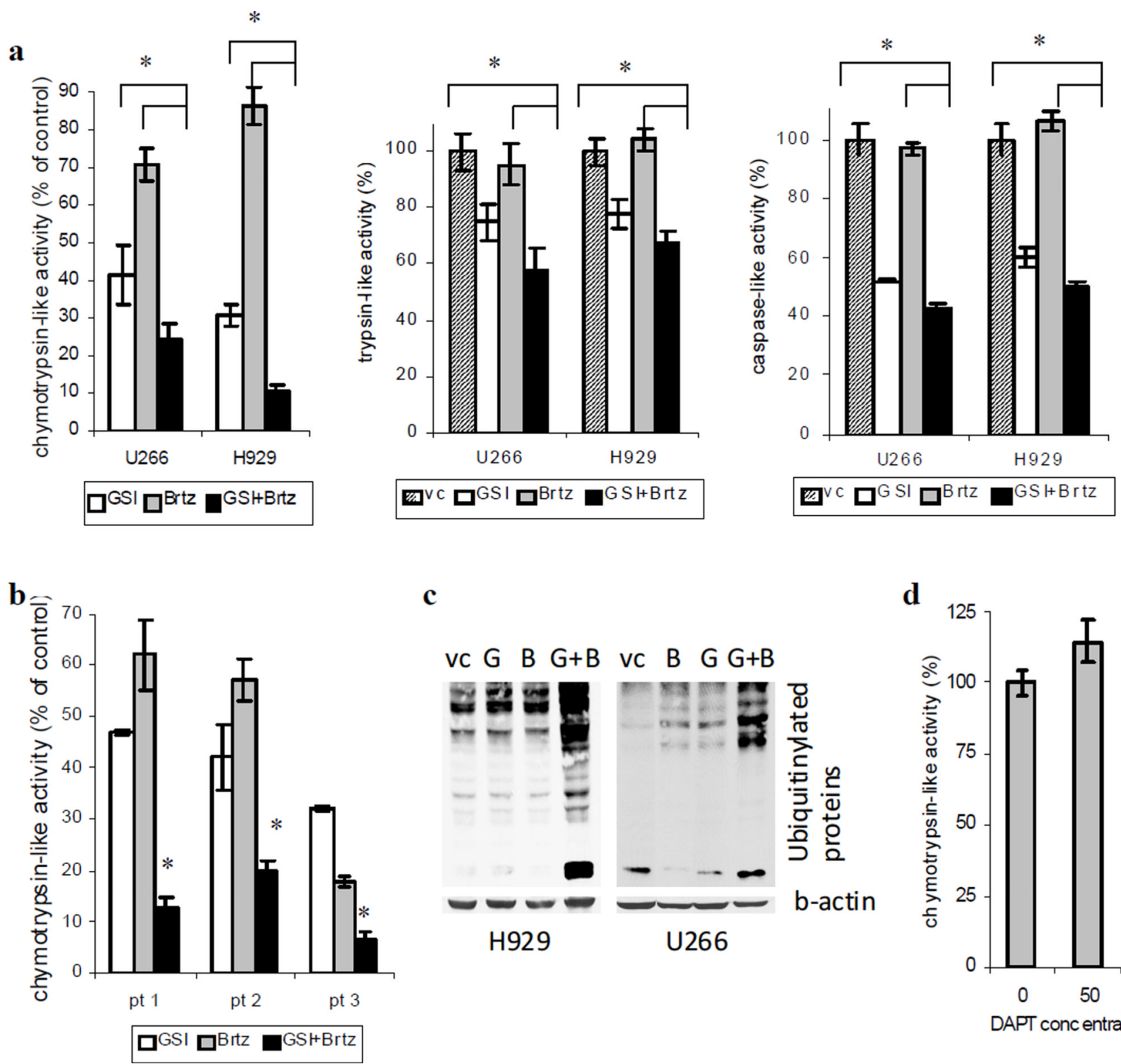

c
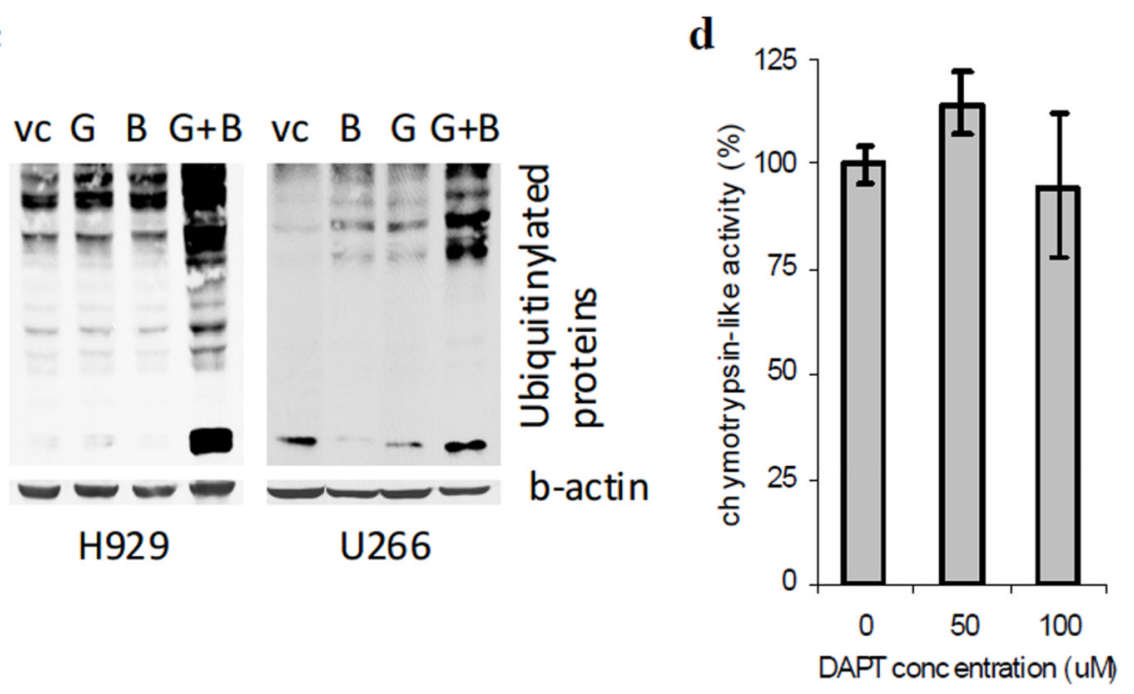

Fig. 4. GSI-XII synergizes with bortezomib in the inhibition of proteasome activity in MM cells H929 or U266 MM cell lines (a) or primary CD138+ MM cells isolated from the bone marrow of patients (pt) with MM (b) were treated with GSI-XII $(G S I)$, Brtz $(B)$, or the combination thereof for $4 \mathrm{hr}$. For MM cell lines GSI was used in concentration $3 \mu \mathrm{M}$ and bortezomib $2 \mathrm{nM}$. Primary MM cells were treated with $4 \mu \mathrm{M}$ GSI (pt 1 and pt 2) or $5 \mu \mathrm{M}$ GSI (pt 3) and $2 \mathrm{nM}$ bortezomib (pt 1 and pt 2) and $5 \mathrm{nM}$ bortezomib (pt 3). Indicated proteasome activities were measured using the corresponding Proteasome-Glo cell-based assay kits (Promega). * - significant difference $(\mathrm{p}<0.05)$ between indicated single-drug treated and the drug combination groups. (c) MM cell lines were treated with GSI, bortezomib $(B)$ or the drug combination for overnight. After that time cells were collected and the level of ubiquitinylated proteins was evaluated by western blotting using specific antibody. Equal loading was confirmed by re-probing the membranes with antibody against beta-actin. (d) H929 cells were treated with DAPT for 4 hr. Proteasome activity was measured using the Proteasome-Glo Chymotrypsin-Like cell-based assay kit (Promega). * 
indicates significant difference $(\mathrm{p}<0.02)$ between the drug combination and single-drug treated groups 
$\mathbf{a}$

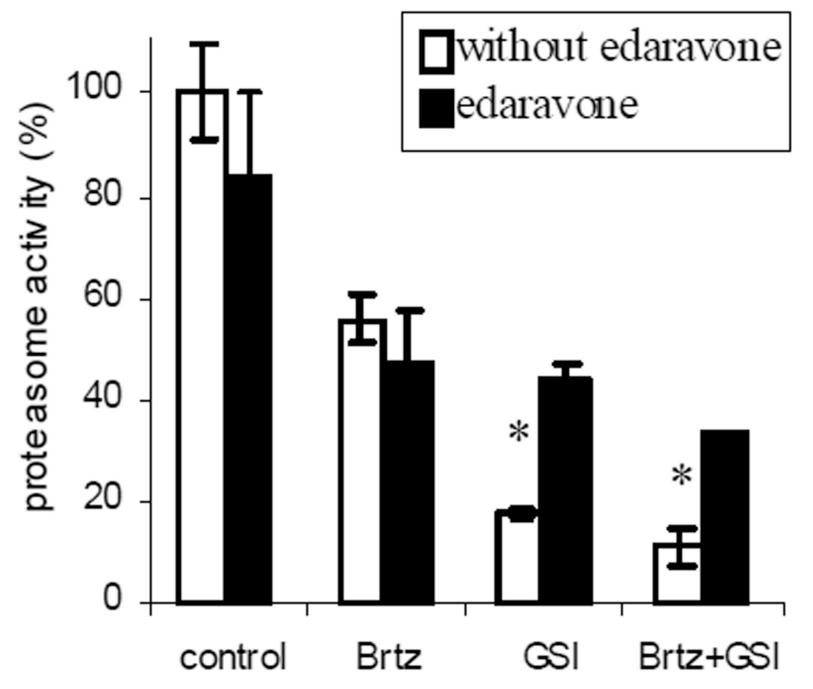

b

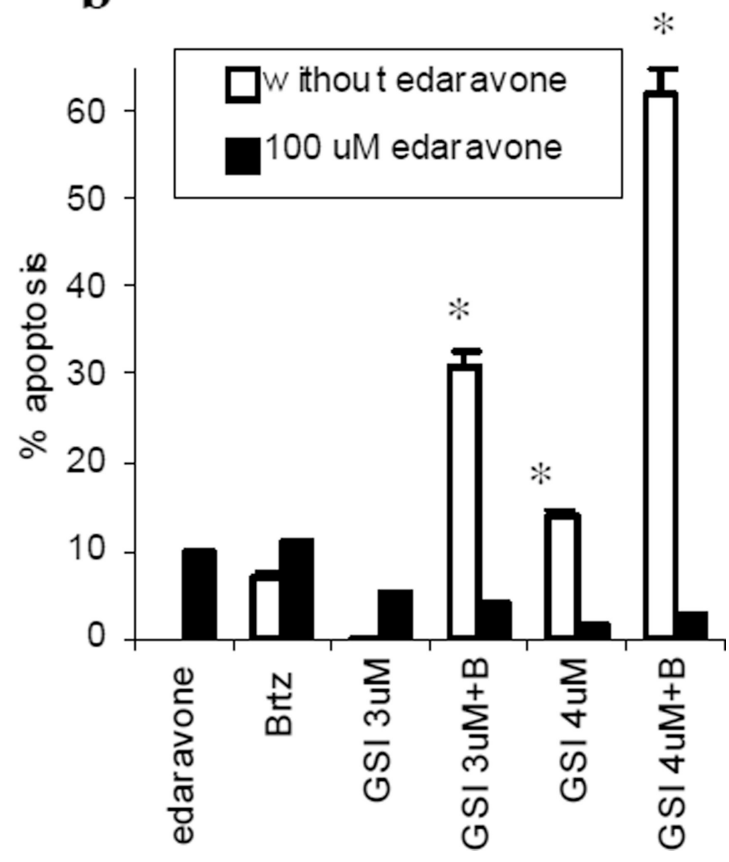

Fig. 5. Effect of the GSI-XII/bortezomib combination on MM cells is abrogated by edaravone treatment

H929 cells were treated with $2 \mathrm{nM}$ bortezomib (Brtz, B), $3 \mu \mathrm{M}$ GSI-XII (GSI) or the drug combination with or without $100 \mathrm{uM}$ edaravone. (a) Proteasome activity was measured using the Proteasome-Glo Chymotrypsin-Like cell-based assay kit after $6 \mathrm{hr}$ of treatment. (b) Apoptosis was detected after $24 \mathrm{hr}$ of treatment by Annexin V-FITC/DAPI staining using a LSR II flow cytometer. * - indicates significant difference $(\mathrm{p}<0.05)$ between groups treated with or without edaravone 